\title{
OSCILLATORY AND PERIODIC SOLUTIONS OF DELAY DIFFERENTIAL EQUATIONS WITH PIECEWISE CONSTANT ARGUMENT
}

\author{
A. R. AFTABIZADEH, JOSEPH WIENER, AND JIAN-MING XU
}

\begin{abstract}
Oscillatory and periodic solutions of retarded functional differential equations are investigated. The study concerns equations with piecewise constant arguments which found applications in certain biomedical problems.
\end{abstract}

1. The study of oscillatory solutions of differential equations with deviating arguments has been the subject of many recent investigations. Of particular importance, however, has been the study of oscillations which are caused by the deviating arguments and which do not appear in the corresponding ordinary differential equation, see $[\mathbf{1}, \mathbf{4}-\mathbf{1 1}, \mathbf{1 3}]$.

In this paper we study oscillatory properties of solutions of the linear delay differential equations with piecewise constant deviating argument of the type

$$
y^{\prime}(t)+a(t) y(t)+b(t) y([t-1])=0,
$$

where $a(t)$ and $b(t)$ are continuous functions on $[0, \infty)$, and $[\cdot]$ designates the greatest integer function. Such equations are similar in structure to those found in certain "sequential-continuous" models of disease dynamics as treated by Busenberg and Cooke [2]. We give sufficient condition under which equation (1) has oscillatory solutions. We emphasize the fact that our condition is the "best possible" in the sense that when $a$ and $b$ are constants the condition reduces to $b>a e^{-a} / 4\left(e^{a}-1\right)$ which is a necessary and sufficient condition. In case of constant coefficients we find conditions under which oscillatory solutions are periodic. As it is customary, a solution is said to be oscillatory if it has arbitrarily large zeros.

2. In this section we give a sufficient condition under which equation (1) has oscillatory solutions.

DEFINITION. A solution of equation (1) on $[0, \infty)$ is a function $y(t)$ that satisfies the initial data $y(0)=C_{0}, y(-1)=C_{-1}$, and the conditions:

(i) $y(t)$ is continuous on $[0, \infty)$;

(ii) the derivative $y^{\prime}(t)$ exists at each point $t \in[0, \infty)$, with the possible exception of the points $[t] \in[0, \infty)$ where one-sided derivatives exist;

(iii) equation (1) is satisfied on each interval $[n, n+1) \subset[0, \infty)$ with integral endpoints.

Received by the editors January 10, 1986.

1980 Mathematics Subject Classification (1985 Revision). Primary 34K10.

Key words and phrases. Oscillations, periodic, functional differential equations:

Research of the first and second authors was partially supported by U.S. Army Research Grant No. DAAG29-84-G-0034. 
THEOREM 1. Suppose that $b(t)>0$, and for $t \geq 0$

$$
\lim _{n \rightarrow \infty} \sup \int_{n}^{n+1} b(t) \exp \left(\int_{n-1}^{t} a(s) d s\right) d t>1
$$

Then the delay differential equation (1) has oscillatory solutions only.

ProOF. We prove that the existence of an eventually positive (or negative) solution leads to a contradiction. For any $k=0,1,2, \ldots$, equation (1) becomes

$$
y^{\prime}(t)+a(t) y(t)+b(t) y(k-1)=0, \quad t \in[k, k+1),
$$

or

(4) $y(k+1) \exp \left(\int_{k}^{k+1} a(s) d s\right)=y(k)-y(k-1) \int_{k}^{k+1} b(t) \exp \left(\int_{k}^{t} a(s) d s\right) d t$.

Now, assume that equation (1) has an eventually positive solution, i.e. $y(t)>0$ for $t>T$, where $T$ is sufficiently large. From (4) for any integer $n \geq[T]+3$, we have

(5) $y(n) \exp \left(\int_{n-1}^{n} a(s) d s\right)=y(n-1)-y(n-2) \int_{n-1}^{n} b(t) \exp \left(\int_{n-1}^{t} a(s) d s\right) d t$, $t \in[n, n+1)$. Since $y(n-2)>0$ and $b(t)>0$, we have

$$
y(n) \exp \left(\int_{n-1}^{n} a(s) d s\right)<y(n-1) .
$$

Also from (4) we have

(7) $y(n+1) \exp \left(\int_{n}^{n+1} a(s) d s\right)+y(n-1) \int_{n}^{n+1} b(t) \exp \left(\int_{n}^{t} a(s) d s\right) d t=y(n)$, $t \in[n, n+1)$. From (6) and (7) we obtain

$$
\begin{aligned}
& y(n)(1-\exp \left.\left(\int_{n-1}^{n} a(s) d s\right) \int_{n}^{n+1} b(t) \exp \left(\int_{n}^{t} a(s) d s\right) d t\right) \\
&>y(n+1) \exp \left(\int_{n}^{n+1} a(s) d s\right) .
\end{aligned}
$$

Since $y(n)$ and $y(n+1)$ are positive,

$$
1>\int_{n}^{n+1} b(t) \exp \left(\int_{n-1}^{t} a(s) d s\right) d t
$$

or

$$
1 \geq \lim _{n \rightarrow \infty} \sup \int_{n}^{n+1} b(t) \exp \left(\int_{n-1}^{t} a(s) d s\right) d t,
$$

which is a contradiction to (2). The proof is the same, in case of existence of an eventually negative solution. Hence, equation (1) has oscillatory solutions only. 


\section{THEOREM 2. Assume that}

(8)

$$
\lim _{n \rightarrow \infty} \inf \left(\exp \left(\int_{n}^{n+1} a(s) d s\right)\right) \cdot \lim _{n \rightarrow \infty} \inf \int_{n}^{n+1} b(t) \exp \left(\int_{n}^{t} a(s) d s\right) d t>\frac{1}{4} .
$$

Then equation (1) has oscillatory solutions only.

ProOF. Suppose that $y(t)>0$ for $t>T$. Let $N=[T]+1$. Then for any integer $n>N$, we have from equation (1), for $t \in[n, n+1)$,

(9) $y(n+1) \exp \left(\int_{n}^{n+1} a(s) d s\right)+y(n-1) \int_{n}^{n+1} b(t) \exp \left(\int_{n}^{t} a(s) d s\right) d t=y(n)$

or

$$
\begin{aligned}
\frac{y(n+1)}{y(n)} \cdot & \frac{y(n)}{y(n-1)} \exp \left(\int_{n}^{n+1} a(s) d s\right) \\
& +\int_{n}^{n+1} b(t) \exp \left(\int_{n}^{t} a(s) d s\right) d t=\frac{y(n)}{y(n-1)} .
\end{aligned}
$$

Let $w_{n}=y(n) / y(n-1)$. Then $w_{n}>0$. We consider two cases:

Case 1. $\lim _{n \rightarrow \infty}$ inf $w_{n}<\infty$. From (10)

$$
\begin{aligned}
\lim _{n \rightarrow \infty} & \inf w_{n+1} \lim _{n \rightarrow \infty} \inf w_{n} \lim _{n \rightarrow \infty} \inf \left(\exp \left(\int_{n}^{n+1} a(s) d s\right)\right) \\
& +\lim _{n \rightarrow \infty} \inf \int_{n}^{n+1} b(t) \exp \left(\int_{n}^{t} a(s) d s\right) d t \leq \lim _{n \rightarrow \infty} \inf w_{n}
\end{aligned}
$$

which is impossible, because, using (8) and the fact that, if $4 A C>1, A>0$, then the inequality $A X^{2}+C \leq X$ has no real solution.

Case 2. $\lim _{n \rightarrow \infty} \inf w_{n}=\infty$. Let $r_{n}=1 / w_{n}=y(n-1) / y(n)$. Then $\lim _{n \rightarrow \infty} \inf r_{n}=0$. From (9) we have

$$
\begin{aligned}
\exp \left(\int_{n}^{n+1} a(s) d s\right)+ & \frac{y(n-1)}{y(n)} \\
& \cdot \frac{y(n)}{y(n+1)} \int_{n}^{n+1} b(t) \exp \left(\int_{n}^{t} a(s) d s\right) d t=\frac{y(n)}{y(n+1)} .
\end{aligned}
$$

This leads us to

$$
0<\lim _{n \rightarrow \infty} \inf \exp \left(\int_{n}^{n+1} a(s) d s\right) \leq 0,
$$

which is a contradiction. So equation (1) cannot have an eventually positive solution. Existence of an eventually negative solution leads to a contradiction. Hence, equation (1) has oscillatory solutions only.

LEMMA 1. Suppose that $y(t)$ is a solution of equation (1) such that $y(n)$ and $y(n+1) \neq 0$ for $n=0,1,2, \ldots, b(t)>0($ or $b(t)<0)$ for $t \geq 0$. Then there exists $a T \in(n, n+1)$ such that $y(T)=0$ if and only if $y(n) \cdot y(n+1)<0$.

PROOF. If $y(n) \cdot y(n+1)<0$, it is clear that there exists $T \in(n, n+1)$ such that $y(T)=0$. We shall show that $y(T)=0$ implies that $y(n) \cdot y(n+1)<0$. For $t \in[n, n+1)$, equation (1) becomes

$$
y^{\prime}(t)+a(t) y(t)+b(t) y(n-1)=0
$$


or

(11) $y(t)=\exp \left(-\int_{n}^{t} a(s) d s\right)\left(y(n)-y(n-1) \int_{n}^{t} b(s) \exp \left(\int_{n}^{s} a(r) d r\right) d s\right)$.

Since the solution $y(t)$ of equation (1) is continuous on $[0, \infty)$, from (11) we get

$$
\begin{aligned}
y(n+1)= & \exp \left(-\int_{n}^{n+1} a(s) d s\right) \\
& \cdot\left(y(n)-y(n-1) \int_{n}^{n+1} b(s) \exp \left(\int_{n}^{s} a(r) d r\right) d s\right) .
\end{aligned}
$$

Also from (11) and the fact that $y(T)=0$, we have

$$
y(n)=y(n-1) \int_{n}^{T} b(s) \exp \left(\int_{n}^{s} a(r) d r\right) d s .
$$

Let us assume that $b(t)>0$. Then from (13), $y(n) \cdot y(n-1)>0$. It is easy to see that, from (12) and (13), $y(n+1) \cdot y(n-1)<0$, and therefore $y(n) \cdot y(n+1)<0$. If $b(t)<0$, then we obtain the same result. The proof is complete.

3. If $a(t)$ and $b(t)$ are constants, i.e.

$$
y^{\prime}(t)+a y(t)+b y([t-1])=0
$$

then condition (8) becomes

$$
b>a e^{-a} / 4\left(e^{a}-1\right) .
$$

We show that (15) is a sharp condition. It is known [3] that (14) with $y(0)=C_{0}$ and $y(-1)=C_{-1}$ has a unique solution given by

$$
y_{n}(t)=C_{n} e^{-a(t-n)}+\frac{b}{a}\left(e^{-a(t-n)}-1\right) C_{n-1}
$$

for $t \in[n, n+1), n=0,1,2, \ldots$, and

$$
C_{n}=\frac{1}{\lambda_{1}-\lambda_{2}}\left(\lambda_{1}^{n+1}\left(C_{0}-\lambda_{2} C_{-1}\right)-\lambda_{2}^{n+1}\left(C_{0}-\lambda_{1} C_{-1}\right)\right)
$$

where $\lambda_{1}, \lambda_{2}$ are the roots of

$$
\lambda^{2}-e^{-a} \lambda+\frac{b}{a}\left(1-e^{-a}\right)=0 .
$$

If these roots are real, assume $\lambda_{1}>\lambda_{2}$.

THEOREM 3. Equation (14) has no oscillatory solution if any one of the following hypotheses holds true:

(i) $b<0$ and $C_{0}-\lambda_{2} C_{-1} \neq 0$; or

(ii) $0<b<a e^{-a} / 4\left(e^{a}-1\right)$ or

(iii) $b=a e^{-a} / 4\left(e^{a}-1\right)$.

ProOF. Any one of the conditions (i)-(iii) implies that (18) has real roots.

If condition (i) holds true, then from (17) we have

$$
\frac{C_{n}}{C_{n-1}}=\frac{\lambda_{1}\left(C_{0}-\lambda_{2} C_{-1}\right)-\left(C_{0}-\lambda_{1} C_{-1}\right) \lambda_{2}\left(\lambda_{2} / \lambda_{1}\right)^{n}}{\left(C_{0}-\lambda_{2} C_{-1}\right)-\left(C_{0}-\lambda_{1} C_{-1}\right)\left(\lambda_{2} / \lambda_{1}\right)^{n}} .
$$


Since $\left|\lambda_{2} / \lambda_{1}\right|<1$ and $C_{0}-\lambda_{2} C_{-1} \neq 0$, then $\lim _{n \rightarrow \infty} C_{n} / C_{n-1}=\lambda_{1}>0$. Hence, $C_{n} / C_{n-1}>0$ for $n>N$. This shows that $C_{n} \cdot C_{n-1}>0$ for $n>N$. Then Lemma 1 shows that there is no $t \in(n, n+1)$ such that $y(t)=0$. So equation (14) has no oscillatory solution. If condition (ii) holds, then $\lambda_{1}>\lambda_{2}>0$ and the same argument shows that $C_{n} \cdot C_{n+1}>0$.

If (iii) holds, then $\lambda_{1}=\lambda_{2}=e^{-a} / 2$, and (17) becomes

$$
C_{n}=\left((n+1) C_{0}-n C_{-1} \lambda_{1}\right) \lambda_{1}^{n} .
$$

If $C_{0}=C_{-1} \lambda_{1}$, then $C_{n}=C_{-1} \lambda_{1}^{n+1}$, which implies $C_{n} \cdot C_{n+1}>0$. If $C_{0} \neq C_{-1} \lambda_{1}$, then

$$
\frac{C_{n}}{C_{n-1}}=\frac{n\left(C_{0}-C_{-1} \lambda_{1}\right)+C_{0}}{n\left(C_{0}-C_{-1} \lambda_{1}\right)+C_{-1}} \lambda_{1}
$$

or $\lim _{n \rightarrow \infty} C_{n} / C_{n-1}=\lambda_{1}>0$, so $C_{n} \cdot C_{n-1}>0$ for $n>N$. Therefore in either case $C_{n} \cdot C_{n+1}>0$, which shows that equation (14) has no oscillatory solution.

REMARK 1 . If $b<0$ and $C_{0}=\lambda_{2} C_{-1}$, then $C_{n}=C_{-1} \cdot \lambda_{2}^{n+1}$. Since $\lambda_{2}<0$, this shows that $C_{n} \cdot C_{n+1}<0$. Therefore in this case equation (14) has oscillatory solutions.

COROLlARY 1. A necessary and sufficient condition for the solutions of equation (14) to be oscillatory is either (15) or

$$
b<0 \text { and } C_{0}=\lambda_{2} C_{-1} .
$$

THEOREM 4 [3]. The solution $y=0$ of equation (14) is asymptotically stable as $t \rightarrow+\infty$ if and only if the moduli of the roots of equation (18) satisfy the inequalities $\left|\lambda_{1}\right|<1$ and $\left|\lambda_{2}\right|<1$.

THEOREM 5. If

$$
a e^{-a} / 4\left(e^{a}-1\right)<b<a e^{a} /\left(e^{a}-1\right),
$$

then every oscillatory solution of (14) tends to zero as $t \rightarrow \infty$. Furthermore, if

$$
-a<b<0 \text { and } C_{0}=C_{-1} \cdot \lambda_{2} \text {, }
$$

then every oscillatory solution of (14) tends to zero as $t \rightarrow \infty$.

LEMMA 2. The solution of equation (14) is periodic of period $k$ if and only if $C_{k-1}=C_{-1}$ and $C_{k}=C_{0}$.

ProOF. Assume that $y(t)$ is periodic of period $k$. Then $y(t+k)=y(t)$ for $t \in(-\infty,+\infty)$. This implies that $C_{k-1}=C_{-1}$ and $C_{k}=C_{0}$. Now, assume that $C_{k-1}=C_{-1}$ and $C_{k}=C_{0}$. From (16) we get

$$
y_{0}(t)=C_{0} e^{-a t}+\frac{b}{a}\left(e^{-a t}-1\right) C_{-1}, \quad 0 \leq t<1
$$

and

$$
y_{k}(t)=C_{k} e^{-a(t-k)}+\frac{b}{a}\left(e^{-a(t-k)}-1\right) C_{k-1}, \quad k \leq t<k+1 .
$$

We see that $y_{0}(t)=y_{k}(t)$, and, because of continuity, $y_{0}(1)=C_{1}=y_{k}(k+1)=$ $C_{k+1}$. Also

$$
y_{1}(t)=C_{1} e^{-a(t-1)}+\frac{b}{a}\left(e^{-a(t-1)}-1\right) C_{0}, \quad 1 \leq t<2
$$


and

$$
y_{k+1}(t)=C_{k+1} e^{-a(t-k-1)}+\frac{b}{a}\left(e^{-a(t-k-1)}-1\right) C_{k}, \quad k+1 \leq t<k+2 .
$$

This shows that $y_{1}(t)=y_{k+1}(t)$. Continuing this way, we can show that $y_{k-1}(t)=$ $y_{2 k-1}(t)$. So $y(t)$ is periodic of period $k$.

THEOREM 6. Let $b>0$. Then every oscillatory solution of equation (14) is periodic of period $k$ if and only if

$$
b=\frac{a e^{a}}{e^{a}-1} \quad \text { and } \quad a=-\ln \left(2 \cos \frac{2 \pi m}{k}\right),
$$

where $m$ and $k$ are relatively prime and $m=1,2, \ldots,[(k-1) / 4]$.

PROOF. Let us assume that equation (14) has periodic solution of period $k$. Then $C_{k}=C_{0}$ and $C_{k-1}=C_{-1}$. Hence, from (17) we have

$$
\begin{gathered}
\left(\lambda_{1}^{k+1}-\lambda_{2}^{k+1}-\lambda_{1}+\lambda_{2}\right) C_{0}-\lambda_{1} \lambda_{2}\left(\lambda_{1}^{k}-\lambda_{2}^{k}\right) C_{-1}=0 \\
\left(\lambda_{1}^{k}-\lambda_{2}^{k}\right) C_{0}-\left(\lambda_{1}^{k} \lambda_{2}-\lambda_{1} \lambda_{2}^{k}+\lambda_{1}-\lambda_{2}\right) C_{-1}=0
\end{gathered}
$$

or $\left(\lambda_{1}^{k}-1\right)\left(\lambda_{2}^{k}-1\right)\left(\lambda_{1}-\lambda_{2}\right)^{2}=0$. Since $b>0$ and the solution is oscillatory, by Corollary $1, \lambda_{1}$ and $\lambda_{2}$ are complex. So $\lambda_{1}^{k}=1$ and $\lambda_{2}^{k}=1$ implies that $\lambda_{1}=e^{2 \pi m i / k}, \lambda_{2}=e^{-2 \pi m i / k}$. But from (18)

$$
\frac{b}{a}\left(1-e^{-a}\right)=1 \quad \text { and } \quad \cos \frac{2 \pi m}{k}=\frac{1}{2} e^{-a}
$$

which is (23). Now assume that (23) is satisfied; we show that every solution is periodic of period $k$. Using (18) and (23) we get

$$
\lambda=\cos \frac{2 \pi m}{k} \pm i \sin \frac{2 \pi m}{k} .
$$

A simple calculation shows that $C_{k}=C_{0}$ and $C_{k-1}=C_{-1}$.

THEOREM 7. Let $b<0$ and $C_{0}=C_{-1} \lambda_{2}$. Then every oscillatory solution is periodic of period 2 if and only if

$$
b=-\frac{a\left(e^{a}+1\right)}{e^{a}-1} .
$$

COROLLARY 2. If $b>0$, then for given $C_{0}$ and $C_{-1}$, the set of all equations of type (14) having periodic solutions is countable.

REMARK 2. The above results were obtained with the implicit assumption $a \neq 0$. If $a=0$, then condition (23) becomes

$$
b=\lim _{a \rightarrow 0} \frac{a e^{a}}{e^{a}-1}=1 .
$$

In this case equation (14) with $a=0$ and $b=1$ has periodic solutions of period 6 . 


\section{REFERENCES}

1. A. R. Aftabizadeh and Joseph Wiener, Oscillatory properties of first order linear functional differential equations, Appl. Anal. 22 (1985), 165-187.

2. S. Busenberg and K. L. Cooke, Models of vertically transmitted diseases with sequential-continuous dymamics, Nonlinear Phenomena in Mathematical Sciences (V. Lakshmikantham, Ed.), Academic Press, New York, 1982, pp. 179-187.

3. K. L. Cooke and J. Wiener, Retarded differential equations with piecewise constant delays, J. Math. Anal. Appl. 99 (1984), 265-297.

4. Y. Kitamura and T. Kusano, Oscillation of first order nonlinear differential equations with deviating argument, Proc. Amer. Math. Soc. 78 (1980), 64-68.

5. T. Kusano and H. Onose, Oscillations of functional differential equations with retarded argument, J. Differential Equations 15 (1974), 269-277.

6. G. Ladas, Sharp conditions for oscillations caused by delays, Applicable Anal. 9 (1979), 93-98.

7. G. Ladas, V. Lakshmikantham, and J. S. Papadakis, Oscillations of higher order retarded differential equations generated by retarded argument, Delay and Functional Differential Equations and Their Applications, Academic Press, New York, 1972, pp. 219-231.

8. G. Ladas and I. P. Stavroulakis, Oscillations caused by several retarded and advanced arguments, J. Differential Equations 44 (1982), 134-152.

9. __ On delay differential inequalities of first order, Funkcial. Ekvac. 25 (1982), 103-113.

10. G. S. Ladde, Oscillations caused by retarded perturbations of first order liner ordinary differential equations, Atti Accad. Naz. Lincei Rend. Cl. Sci. Fis. Mat. Nat. 63 (1977), 351-359.

11. H. Onose, Oscillatory properties of first order differential inequalities with deviating argument, Funckial. Ekvac. 26 (1983), 189-195.

12. S. M. Shah and J. Wiener, Advanced differential equations with piecewise constant argument deviatiors, Internat. J. Math. Math. Sci. 6 (1983), 671-703.

13. B. Singh, Necessary and sufficient condition for maintaining oscillations and nonoscillations in general functional equations and their asymptotic properties, SIAM J. Math. Anal. 10 (1979), 18-31.

Department of Mathematics, Ohio University, Athens, Ohio 45701 (Current address of A. R. Aftabizadeh and Jian-Ming Xu)

Department of Mathematics and Computer SCIEnCE, PAN AMERiCAN UniverSITY, EDINBURG, TEXAS 78539 (Current address of Joseph Wiener) 